\title{
MODELING, PROGRAMMING AND SIMULATION OF ROBOTIZED WORKCELLS CREATED FOR INDUSTRIAL AND SERVICE NEEDS
}

\author{
Roman Trochimczuk $^{1}$, Andrzej Lukaszewicz ${ }^{1}$, Ryszard Szczebiot ${ }^{2}$, Alexey G. Kirillov ${ }^{3}$, Ile Mircheski ${ }^{4}$ \\ ${ }^{1}$ Bialystok University of Technology, Poland; ${ }^{2}$ Lomza State University of Applied Sciences, Poland; \\ ${ }^{3}$ Vitebsk State Technological University, Belarus; ${ }^{4}$ Ss Cyril and Methodius University in Skopje, \\ Republic of Macedonia \\ r.trochimczuk@pb.edu.pl,a.lukaszewicz@pb.edu.pl, rysbiot@pwsip.edu.pl, \\ kirillov.malp@gmail.com, ile.mircheski@mf.edu.mk
}

\begin{abstract}
The paper shows and compares the functional features of off-line software environments of selected manufacturers of industrial robots. These softwares used for modelling, programming and simulation of robotic workcells are created for industrial and service needs (e.g. agricultural services), or simulation use of robots adapted to cooperate with humans. The use of such solutions greatly improves the assessment of the safety and exploitation conditions of robotic systems in various areas and aspects of life. It gives the investor an opportunity to make an opinion on the economic and social aspects of using robots in the manufacturing and service sector, too. Such systems (in particular Festo COSIMIR ${ }^{\circledR}$ and ROS programs described in the paper) are becoming an important element of the so-called Industry 4.0, so you should have knowledge about them.
\end{abstract}

Keywords: workcell, robotization, programming of robots, modeling and simulation.

\section{Introduction}

The implementation of robots in industrial production systems and the services sector, significantly affects the achievement of the highest level of automation by the factories, manufacturing and service companies and the local producers on the agricultural market. Products manufactured by using robotic systems are characterized by high repeatability and precision of manufacturing. Indeed, this increases the final competitiveness of the product on the market. The replacement of farm workers by robots in daily, repetitive tasks that serve the production process (for example, the use of milking robots, the use of robots for dosing feed, or the use of robots cleaning the grates of slurry floors from impurities in cattle farming etc.) translates into transparency and increased production efficiency [1-4]. It also gives the possibility of variability of manufactured products. The time saved can be used to increase the scope of current production. Higher work efficiency, simplified management of the control process resulting from robotization also significantly increase the chances of local producers in global economic competition [5]. The product manufactured by robots is of higher quality due to the increase in the standard of the manufacturing process and the possibility of carrying out more tests. Do not forget about removing employees from a risky and onerous work environments.

Automation and robotization applied in various areas of human activity has meant that, together with the exclusion of low-skilled employees, who have been performing repetitive, everyday operation, there has also been a need to create new jobs. Jobs are mainly associated with the employment of educated engineers. Engineers are responsible mainly for service and the current support of the control and safety systems, for maintenance of the components of the robotic work cells, for retrofitting the robot system in case of variation of production, for adjustment of the robot control software to the current production needs [6]. From the point of view of daily operation of the robotic system, it is necessary to provide the supervising persons with the relevant solutions with appropriate programming tools. These tools are to enable synchronization of robot movements depending on the required sequence of operations, while maintaining safety conditions. The aim is to use such software tools for modeling, programming and simulation of robotic work cells [7-10]. Exploring the possibilities of cooperation between man and robot and the other devices, the possibility of eliminating failures and reporting, current robot system diagnostics together with the risk assessment of collisions between the robot and other components of the workcell are features that are part of the current trend of development of such simulation programs [11-13].

The functionality of selected programming environments for programing and simulation of robotic workcells will be defined in the paper. The starting point is the experience of the authors and their own thoughts on a systematic approach to the issues of industrial and service robots. By presenting the possibilities of various programs supporting the work of automation and robotics engineers, in the field of modeling, programming and simulation of robotics workcell, one of the possible ways to 
educate future designers and the ways to increase investor awareness will be shown. Due to this knowledge, future investors will be able to form an opinion on the economic and social aspects of the use of robots in the manufacturing and service sector as well as on the scale and technical needs of the newly planned enterprise.

\section{Materials and methods}

Permanent shortages in employing qualified personnel mainly contribute to the introduction of the Industry 4.0 assumptions and solutions in various industry and service areas [14-15]. Thanks to this approach, you can dynamically and flexibly react to meet the changing needs of the market. Investment in robotic technologies and building new business models in the future will translate into higher profits and the value of the company, despite the high initial investment in such technologies of manufacturing.

One of the many available tools supporting virtual modeling, programming and testing the concept of robotics workcell is dedicated software environment to a specific robot. Currently each of the important manufactures of robots in the world has to offer simulation software to support its technical solution [16]. The names of the main manufacturers of robots and their technical products supporting the solutions offered are summarized in Table 1.

Table 1

List of manufacturers of robots and dedicated simulation products

\begin{tabular}{|c|c|c|}
\hline Manufacturer & $\begin{array}{c}\text { Name of the product for modeling, } \\
\text { programming and simulation }\end{array}$ & Programming language \\
\hline Kuka Roboter & Kuka Sim Pro & KRL \\
\hline Fanuc Robotics & RoboGuide & KAREL \\
\hline Mitsubishi Robotics & RT Toolbox & MELFA-BASIC \\
\hline Comau & RoboSim Pro & PDL2 \\
\hline Adept & Adept ACE & $\mathrm{V}, \mathrm{V}+$ \\
\hline $\mathrm{ABB}$ & RobotStudio & RAPID \\
\hline Kawaski Robotics & K-Roset & $\mathrm{AS}$ \\
\hline Stäubli Robotics & Staubli Robotics Suite & VAL3 \\
\hline Universal Robots & RoboDK & URScript \\
\hline Epson & Epson RC + & $\mathrm{SPEL}^{+}$ \\
\hline Denso Robotics & WinCAPS III & PacScript \\
\hline $\begin{array}{l}\text { Motoman Robotics- } \\
\text { Yaskawa }\end{array}$ & MotoSim EG-VRC & INFORM \\
\hline Panasonic-Matsushita & $\begin{array}{l}\text { DTPS Desk Top Programming \& } \\
\text { Simulation System }\end{array}$ & PARL-1 \\
\hline Toshiba & TSAssist & SCOL \\
\hline FESTO & $\begin{array}{c}\text { COSIMIR }^{\circledR} \text { (Cell Oriented } \\
\text { Simulation of Industrial Robots) }\end{array}$ & $\begin{array}{l}\text { FTL or different depending on } \\
\text { the used robot type }\end{array}$ \\
\hline
\end{tabular}

Analyzing the usability of each of the commercial programs mentioned in Table 1, their functional features can be reduced to functionality in the full planning of the system robot - work cell, controlling the position achieved by the components system, writing programs for robots, optimizing the distribution of workcells, simulations and visualization of them. All movements of robots and devices interacting with them, e.g. feeding and receiving systems from/to the robot (including conveyor belts, dial-indexing tables, gravity magazines and other specialized positioners), operation of elements controlling the work of solutions (e.g. sensor systems for recognizing of the color of workpiece, the distance and presence sensors etc.), simulation of the digital inputs and outputs of the robot controller or the entire work cell can be fully simulated. The simulation may simultaneously take into account the conditions of time optimization of the work cycle, the scope of the equipment working space, with the collision detection between elements of the robotic system and determining the path of the end effector's movement in three-dimensional space. This allows to eliminate most program errors already at the stage of virtual simulation of the robot system. 
All of the analyzed environments supporting the work of a robotics engineer allowed for full simulation of the functionality of the teach pendant dedicated to a given robot. Thanks to this, you can control a virtual robot in Teach-In mode, automatically generate and read the current position of the robot arm and create a position list necessary to write a control program. You can also change the speed of the robot in simulation and learning mode, run/stop motors of the robot and calibrate procedure of the other elements of the robot system; it is possible to simulate emergency situations and develop ways to return from the state of failure to the standard operating state. Developed programs after the testing phase in a virtual environment can be directly sent to the control system of the real robot. For the purpose of the developed program you can also read the current states from the real PLC controller of an industrial robot. Programs usually allow entering the data necessary to establish factory settings of the real robot. This option is often used during periodic service of the device, e.g. in situation when we need replacing the battery supporting the robot's logic.

For modelling the virtual components of the proposed workcell commonly CAD systems are used (e.g. Catia, SolidWorks, Solid Edge, Autodesk Inventor) [17]. The solid objects created in these CAD environments can be imported into the off-line software [18;19] used for programming the selected robot. Such a file that it can be correctly imported and used for simulation in robot programing environments must be saved in the appropriate file format, e.g. STL-file. The functionality of the CAD environments allows for professional creation of three-dimensional models of objects that are the basis for the design of a new project of a work cell, or for the modernization of the existing solution while maintaining their real form and dimensions [20;21].

The library of models implemented in the robot programming environments can be used to modeling the system robot - workcell. They contain ready-made solutions offered by a given manufacturer, e.g. basic forms of end effectors, conveyor belts, part feeders, etc. Operation of these components is realistically simulated without additional software. Sometimes in these environments there is the library of solid primitives (e.g. prism, cube, cylinder, sphere). With them it is possible to create elements of the workcell. You can also use them to supplement the created 3D models of workcell components [22]. Helpful in modeling process is additional texture library, used to give the real appearance of the components of the robot system.

Most of the available software on the market support the programming and simulation of industrial robots under the Microsoft Windows operating system. Manufacturers also support software solutions for the Linux operating system. Under Linux system, the development of computer programs is also open-source license. An example of such a development environment is ROS (Robot Operating System - collection of software frameworks for robot software development) [23;24].

Typical properties of the Microsoft Windows system, i.e. the size of open windows, the drag and drop method, context menu and clipboard function greatly simplify and speed up the programming, modeling and simulation process. When it comes to simulating the operation of the robotic workcell, such programs are quite demanding. The programs require proper operation of the appropriate amount of memory and multi-core microprocessor. For this reason, the simulation of the project to a certain maximum number of robots is usually limited, e.g. a workcell with up to ten robots. The requirements for graphics card parameters are not so restrictive in this case.

Without any major problems in each of the analyzed software you can print the created program with the corresponding list of positions and graphical representation of the work cell. The preview of the designed service or industrial process is possible from different views. The user can simultaneously track the current positions of the robot arm, in a local or global coordinate system, reading the rotation and translation displacements. There is also the option of reading a control program written in a higher level programming language (the programming language is selected depending on the type of the used robot) [25].

Regardless of the choice of the programming language of the robot, the main syntax of the program is usually based on commands defining variables, program blocks, control operators (conditional instructions), action operators, macro definitions, procedures and commands that enable synchronization of the robot activities, command definition of object coordinate systems in threedimensional space. Most of the programs have wizards to help programing the typical tasks for the robot, e.g. a palletization task, or a pick-and-place task. Programming of a robot and the workcell 
consists in filling the positions list assigned to the robot, remembering successive movements of the robot arms, manipulating the processed object, moving and grasping other objects, definitions of operation of peripheral devices. The TEACH-IN function can be used for this purpose, which is very helpful in the implementation of the operations assumed by the designer. An important element of the control program is the possibility to correct the introduction of the index values, the outputs are used to control the robot end effector. The final trajectories of the robot's arm are defined as the position and orientation of the TCP (Tool Center Point), relative to the tool or the workcell. The result of such generated trajectory is the optimized robot movement, the ability to evaluate the robot's working space and determination of the relative speed of operation.

To simulate the operation of the robot workcell modeled and programmed objects should be merged into one. The created program should be loaded into the controller of the simulated real robot. The user, when choosing simulation parameters, must decide whether he wants to carry out the work cell simulation with fixed cycle (fixed cycle - the difference resulting from the time between refreshing the graphics window of the workcell), or if it has to be a dynamic simulation (achievement in real-time synchronization between the simulated and actual time). After setting these parameters, the loaded program will start to be simulated step by step, and the counter will inform the user about the duration of the simulation.

By using the robot's learning window interface, you can influence individual movements of robot links by simulating its movement. The main program window usually contains a list of all elements of the workcell, including objects and associated materials, as well as properly defined connections of the robot controller inputs/outputs or other workcell elements.

Most of the analyzed programs supporting the process of programming, modeling and simulation of robotic workcells also have various modules extending the simulation capabilities (e.g. collision detection module, sensor and process simulation module, trajectory generation module, PLC simulation module, etc.). Such modules significantly improve the comfort and efficiency of the design robotic workcell, and thus the process of modeling, programming and simulation becomes very realistic. It is possible to predict and assess the risk of collision, thus eliminating the cause, extending the simulation conditions by parameterizing the sensors, interpreting PLC programs, editing and optimizing programs, as well as automatically generating the trajectory of motion.

\section{Results and discussion}

1. The main problem that can be encountered by an engineer at the stage of building a project and testing it with the use of computer tools is the inability to integrate robotic solutions of different manufacturers in one project. The robot manufacturer supports only his solutions and gives him a dedicated tool that is incompatible with competing products. This problem can be solved using products similar to the Festo COSIMIR ${ }^{\circledR}$ program. This program gives you the opportunity to use the robotic libraries of the following manufacturers: ABB, Adept, Fanuc, Kuka, Manutec, Mitsubishi, Niko, Reis, Stäubli, VW and extensive equipment libraries, in which various types of end effectors are included. It allows programming of robots in language versions IRL (Industrial Robot Language), BAPS (Bosch Automatisierungs - Programmier Sprache), MRL (Mitsubishi Robot Language), SPRL (Simple Robot Programming Language), MELFA Basic III, RAPID, KRL (Kuka Robot Language) [26, 27].

2. Robotics middleware ROS (Robot Operating System) open-source product does not impose any restrictions as to the type and make of the supported robot arm, or the project. Leading manufacturers also support the environment with hardware (ABB, Adept, Comau, Fanuc, Kuka, Motoman, Universal Robots). The main problem in using this tool stems from the fact that we alone need to create an interface to program and visualize the operation of the designed workcell solution. To create this, you need additional designer skills that can affect the final decision of the investor.

3. The use of programs only written and simulated on the computer for more complex tasks, e.g. welding, applying paint coatings, polishing, dispensing glue and usually is not enough. This is due to the fact that besides the simulation itself, the engineer must perform extensive time trials on the actual work cell objects in order to be able to gradually optimize the parameters assigned to the 
process, for example, minimizing the amount of the paint used, measuring the energy consumption, and qualitative evaluation of the final product.

4. The main arguments that will appeal the use of software for visualization and programming of industrial and service robots are:

- increasing productivity by simulating the operation of a robotic system by means of a computer, by which the downtime on the real production line is reduced,

- opportunity to optimize the movement of the robot relative to the cycle and the quality of the work performed, before loading the program to the real robot control system,

- ability to prevent collisions of the robot arm and the entire system,

- possibility of printing the development program with the corresponding positions list and graphical representation of the workcell.

\section{Conclusions}

1. Nowadays, automation and robotization of manufacturing processes does not apply only to large enterprises. Increasingly, small and medium-sized enterprises are forced by the market to reduce production costs, while increasing the product quality, through the use of flexible, rational methods of robotic production. Thanks to these the manufacturer can quickly respond to the market needs by adjusting the product to the current demand, as well as increasing the chances to achieve commercial success.

2. Each robot manufacturer has in its offer its own application supporting the process of modeling the workcell, its programming and virtual simulation of the robot. The main problem with using this type of programs appears in a situation when in the project robots of different manufacturers are used, each of which has a different specificity of programming. Using the Festo Cosimir ${ }^{\circledR}$ and ROS programs solves this problem. These tools allow programming and optimizing the workcell elements. Other similar programs can be used to employee training in systems working, optimizing processes, planning production timetables and testing new solutions.

3. From the point of view of an engineer, computer programs simulating real robotic systems allow you first of all to present the investor with the concept of building a new application, without physical equipment involved in the new the project. Hence, the service becomes more available. The functionality of the programs to simulate the operation of robots, such as the ability of risk assessment, collision detection, estimation of the process performance, optimization of the operation and adjustment of the deployment of the equipment to the size of buildings can convince the investor more about the merits of a significant financial investment in the robotic system.

\section{Acknowledgements}

This paper was performed within the framework of S/WM/1/2016 and S/WM/1/2018, implemented in the Bialystok University of Technology fund from the Polish Ministry of Science and Higher Education. Some ideas were made with the collaboration of researchers from the Lomza State University of Applied Sciences (Poland) and the Vitebsk State Technological University (Belarus) and the Ss Cyril and Methodius University in Skopje (Macedonia).

\section{References}

[1] Edan Y., Han S., Kondo N. Automation in agriculture. In: Springer handbook of automation, Berlin, Springer, 2009, pp. 1095-1128.

[2] Hyde J., Engel P. Investing in a robotic milking system: A Monte Carlo simulation analysis. Journal of Dairy Science, vol. 85, issue 9, 2002, pp. 2207-2214.

[3] Kuzmierowski T., Trochimczuk R. Automation of work in cowsheds using mobile robots with haptic devices supported by virtual zones of influence. Proceeding of 17th International Scientific Conference on Engineering for Rural Development, Jelgava, Latvia, May 23-25, 2018, pp. 976-981.

[4] Libin Z., Qinghua Y., Guanjun B., Yan W., Liyong Q., Feng G., Fang X. Overview of research on agricultural robot in China. International Journal of Agricultural and Biological Engineering, vol. 1 , issue 1,2008 , pp. 12-21. 
[5] Pedersen S.M., Fountas S., Have H., Blackmore B.S. Agricultural robots—system analysis and economic feasibility. Precision agriculture, 7(4), 2006, pp. 295-308.

[6] Mourtzis, D., Doukas M., Bernidaki D. Simulation in manufacturing: Review and challenges. Procedia CIRP, 25, 2014, pp. 213-229.

[7] Mitsi S., Bouzakis K.D., Mansour G., Sagris D., Maliaris G. Off-line programming of an industrial robot for manufacturing. The International Journal of Advanced Manufacturing Technology, 26(3), 2005, pp. 262-267.

[8] Rogalinski P. An approach to automatic robots programming in the flexible manufacturing cell. Robotica, 12(3), 1994, pp. 263-279.

[9] Roßmann, I.J., Lenk, D.I.P. Optimization of Industrial Robot Work Cells using the Automated Variant Simulation. In Proc. of the 7th EUROSIS Int. Industrial Simulation Conf.(ISC), Loughborough, UK, 2009, pp. 133-137.

[10] Hauer S., Malisa V., Hieger C., Stuja, K. Design and simulation of modular robot work cells. Annals of DAAAM \& Proceedings, 2009, pp. 1801-1803.

[11]Balchanowski J., Wudarczyk S. Simulation researches of translational parallel mechanisms. Proceeding on 10th International Conference on the Theory of Machines and Mechanisms Location: Liberec, Czech Republic, Sep 02-04, 2008, pp. 35-40.

[12] Laski P.A., Takosoglu J.E., Blasiak S. Design of a 3-DOF tripod electro-pneumatic parallel manipulator. Robotics and Autonomous Systems, 72, 2015, pp. 59-70.

[13] Megahed S.M., Megahed S.M. Principles of robot modelling and simulation. J. Wiley, 1993.

[14] Lasi H., Fettke P., Kemper H.G., Feld T., Hoffmann M. Industry 4.0. Business \& Information Systems Engineering, vol. 6, issue 4, 2014, pp. 239-242.

[15] Bahrin M.A.K., Othman M.F., Azli N.H.N., Talib M.F. Industry 4.0: A review on industrial automation and robotic. Jurnal Teknologi, vol. 78, 2016, pp. 6-13.

[16] Kaczmarek W., Panasiuk J. Comparison of the method of robot control and programming ABB, FANUC, MITSUBISHI and KUKA, Polish Scientific Publishers PWN, 2017, pp. 270-276.

[17] Łukaszewicz A., Skorulski G., Szczebiot R. The main aspects of training in the field of computeraided techniques (CAx) in mechanical engineering. Proceedings of 17th International Scientific Conf. on Engineering for Rural Development, May 23-25, 2018, Jelgava, Latvia, pp. 865-870.

[18] Miatliuk K., Łukaszewicz A., Siemieniako F. Coordination method in design of forming operations of hierarchical solid objects. Proceedings of International Conference on Control, Automation and Systems: ICCAS'2008, Oct. 14-17, 2008, Seoul, South Korea, pp. 2724-2357.

[19] Poniatowska M., Werner A. Simulation tests of the method for determining a CAD model of freeform surface deterministic deviations. Metrology and Measurement Systems, vol. 19, issue 1, 2012, pp. 151-158.

[20] Trochimczuk R., Prochor P. Finite element analysis of temperature distribution in sport horses' skin during rehabilitation massage. In Proceedings of 16th International Scientific Conference Engineering For Rural Development, 2017, May, pp. 935-940.

[21] Trochimczuk R., et al. Finite element method stiffness analysis of a novel telemanipulator for minimally invasive surgery. Simulation: Transactions of the Society for Modeling and Simulation International, 2019, p.11. DOI: 10.1177/0037549719835920.

[22] Łukaszewicz A., Panas K., Szczebiot R. Design process of technological line to vegetables packaging using CAx tools. Proceedings of 17th International Scientific Conference on Engineering for Rural Development, May 23-25, 2018, Jelgava, Latvia, pp. 871-876.

[23] Quigley M., et al. ROS: an open-source Robot Operating System. In ICRA Workshop on Open Source Software, vol. 3, issue 3.2, 2009, 5 p.

[24] Quigley M., Gerkey B., Smart W.D. Programming robots with ROS: a practical introduction to the Robot Operating System. O'Reilly Media, Inc., 2015. 448 p.

[25]Biggs G., Macdonald B. A survey of robot programming systems. In: Proceedings of the Australasian Conference on Robotics and Automation, 2003. pp. 1-3.

[26] Freund E., Hypki A., Bauer R., Pensky D.H. Real-time coupling of the 3D workcell simulation system COSIMIR. In: Proceeding of International Conference on Information Technology \& Applications (ICITA 2002), Bathurst, Australia, 2002, pp 645-650.

[27] COSIMIR ${ }^{\circledR}$ Getting Started, Short introduction into 3D simulation and offline programming of robot - based workcell with COSIMIR ${ }^{\circledR}$, Festo Didactic GmbH, 2001. 42 p. 